\title{
Theoretical Investigations on Heteronuclear Chalcogen- Chalcogen Interactions: On the Nature of Weak Bonds Between Chalcogen Centers
}

\author{
Christian Bleiholder, ${ }^{[\mathrm{a}],[\mathrm{b}]}$ Rolf Gleiter, ${ }^{[\mathrm{a}] \star}$ Daniel B. Werz, ${ }^{[\mathrm{a}],[\mathrm{c}]}$ and Horst \\ Köppel ${ }^{[\mathrm{d}]}$
}

[a] Organisch-Chemisches Institut der Universität Heidelberg, Im Neuenheimer Feld 270, D-69120 Heidelberg, Germany. e-mail: rolf.gleiter@oci.uni-heidelberg.de

${ }^{[b]}$ Deutsches Krebsforschungszentrum (DKFZ), Im Neuenheimer Feld 280, D-69120 Heidelberg, Germany.

${ }^{[c]}$ Laboratorium für Organische Chemie, Wolfgang-Pauli-Str. 10, Swiss Federal Institute of Technology (ETH) Zürich, ETH-Hönggerberg, $\mathrm{CH}-8093$ Zürich, Switzerland.

[d] Physikalisch-Chemisches Institut der Universität Heidelberg, Im Neuenheimer Feld 229, D-69120 Heidelberg, Germany.

SUPPORTING INFORMATION 


\section{S.1. Detailed algorithm to distinguish between hydrogen bonds and chalcogen- chalcogen-interactions using NBO analysis}

The algorithm to distinguish between hydrogen bonds and chalcogen-chalcogen interactions used in this work is based on the natural bond orbital analysis as provided by the NBO program of the Gaussian03 suite of programs. In the NBO algorithm, first the natural atomic orbitals (NAOs) are computed. Then, some NAOs are linearly combined to obtain natural bond orbitals (NBOs). These NBOs can be grouped into four distinct categories, depending on the number of atoms their constituting NAOs are centered on (in our case either 1 or 2$)$ and their electron occupation number $\rho(\rho \leq 2)$. Bond NBOs (BD) are two-centered, while core (CR), lone pair (LP) and Rydberg (RY) NBOs are centered on one atom only. For each of these occupied stabilizing NBOs, there exists also one destabilizing anti-bonding orbital. In addition to the NBOs themselves, the NBO program also provides the stabilizing interaction energies between pairs of NBOs (always one bonding and one anti-bonding NBO) as computed by second order perturbation theory. By summing up all the interaction energies stemming from two NBOs located on chalcogens in $E_{X-X}($ eq $S 1)$, we can approximate the strength of the interaction taking place between the two chalcogen centers in the model systems 1-32. Analogously, summing up all interaction energy terms having at least one of the interacting NBOs involving a hydrogen into $\mathrm{E}_{\mathrm{H} \text {-bond }}$ (eq S2), we get an approximation on the amount of hydrogen bonding between the two monomers in the model systems 1-32. Interactions between orbitals neither involved in hydrogen bonding nor chalcogen-chalcogen interaction has been summed into an $E_{\text {rest }}$ (eq S3), which was negligible for each aggregate. 
(eq S1) $\quad E_{X-X}=\sum_{N B O S} \varepsilon_{X \rightarrow X}^{(2)}$

(eq S2) $\quad E_{\text {hbond }}=\sum_{N B O s} \varepsilon_{a n y \rightarrow y-H}^{(2)}+\sum_{N B O s} \varepsilon_{y-H \rightarrow a n y}^{(2)}$

(eq S3) $\quad E_{\text {rest }}=\sum_{N B O s} \varepsilon_{\text {any } \rightarrow \text { any }}^{(2)}-E_{X-X}-E_{H b o n d}$

It should be noted here, that the intermolecular interaction energies $E_{\text {int }}$ for the model systems 1-32 as given in Table 2 of this work are in no direct correspondence with the interaction energies obtained by the NBO analysis. Therefore, the actual value of the quantities $E_{X-X}$ or $E_{H-b o n d}$ are of no direct meaning. However, the relative strength of hydrogen-bonding versus chalcogen-chalcogen interaction is readily accessible from it.

\section{S.2. Extending the SAPT calculations to tellurium containing systems}

In our previous investigation on chalcogen-chalcogen interactions (Ref 19) we have performed SAPT calculations on the non-tellurium containing systems only. Towards this end, we have employed the $6-311 \mathrm{G}^{* *}$ basis set and have used the MP2/cc-pVTZ-ECP optimized geometries. The $6-311 \mathrm{G}^{* *}$ basis set for benchmarking showed that it represents a rather good approximation to the cc-pVTZ-ECP basis (See Supporting Information of Ref. 19).

As the exclusion of tellurium containing systems would represent a strong limitation in the view of this work, we have investigated how the DGDZVP basis set would describe the non-covalent interaction occuring in the dimers 1-32. In particular, three criteria had to be 
satisfied in order for us to consider the DGDZVP basis set usable for the SAPT calculations:

1. The trends observed in the interaction energies for the MP2/cc-pVTZ-ECP and $\operatorname{CCSD}(\mathrm{T}) / \mathrm{cc}-\mathrm{pVTZ}$-ECP level of theories must be qualitatively reproduced by the MP2/DGDZVP and CCSD(T)/ DGDZVP level of theories using the optimized geometries of the particular MP2 level of theory.

2. The trends observed in the components of the SAPT/6-311G** calculations must be qualitatively reproduced by the SAPT/DGDZVP calculations for identical geometries.

3. The trends observed in the interaction energies for the MP2/cc-pVTZ-ECP and $\operatorname{CCSD}(T) / c c-p V T Z-E C P$ level of theories must be qualitatively reproduced by the SAPT/DGDZVP level of theory.

The comparison of the total interaction energies $E_{\text {int,MP2 }}^{\text {cc-pVIZ-ECP }}, E_{\text {int,MP2 }}^{\text {DGDVP }}$ as well as $E_{\mathrm{int}, \mathrm{CCSD}(\mathrm{T})}^{\mathrm{DGDZVP}}$ indicates (Figure FS1) that the trends produced by the three distinct levels of theory are similar to each other, although definitely quantitative deviations are observed. However, the trends (e.g. when going from $\mathrm{X}_{2}=\mathrm{O} \rightarrow \mathrm{S} \rightarrow \mathrm{Se} \rightarrow \mathrm{Te}$ in each family) are qualitatively well reproduced in all systems $\mathbf{1}$ - 32 .

The second criteria, i.e. that the SAPT calculations themselves are consistent when using the two basis sets $6-311 G^{* *}$ and DGDZVP, is examined by the help of the Figures FS2 to FS6. In order two investigate this, the same geometry had to be used for both calculations, and towards this end we have used the MP2/cc-pVTZ-ECP optimized ones. Please note that the interaction energies so obtained do not equate those obtained on the DGDZVP optimized geometries and are not being used in this work otherwise than to compare the performance of two basis sets. Additionally, please note that tellurium containing systems 
could not be treated. In the following it is assumed that the trends observed for the remaining model systems are transferable to these systems as well.

It is clearly seen in Figure FS2, that the total interaction energies $E_{\text {int,SAPT }}^{6-311 G^{* *}}$ and $E_{\text {int,SAPT }}^{\text {DGDZVP }}$ do not show significant deviations; the greatest single difference is $0.53 \mathrm{kcal} / \mathrm{mol}$ for system 27. However, this still fits well within the accuracy of the theoretic levels applied here, and the other deviations are even smaller. Comparing the electrostatic contribution to the respective interaction energies (Figure FS3), the only notable deviation between the two basis sets is that for model system 3 the basis set $6-311 \mathrm{G}^{* *}$ yields a slightly anti-bonding character $(0.06 \mathrm{kcal} / \mathrm{mol})$, while DGDZVP gives a bonding character $(0.18 \mathrm{kcal} / \mathrm{mol})$. However, the trend within the family 1, 2, $\mathbf{3}$ is still the same with both basis sets: the electrostatic component becomes less bonding when going from $X_{2}=0$ to $S$ to $S e$. However, this finding indicates that the absolute values of the interaction components should be interpreted with care. For all other computed systems, the trends for the two basis sets are mutually equivalent. The trends for induction, dispersion as well as exchange-correlation components of interaction energies (Figure FS4, FS5 and FS6, respectively) are well reproduced by both methods, although slight quantitative deviations are clearly observed. However, these deviations are in each case well within the accuracy of the applied methods.

It follows from these observations, that by using the SAPT calculation will give analogous results, irrespective of using any of the two basis set DGDZVP and 6-311G**.

The consistency between the SAPT/DGDZVP and MP2/cc-pVTZ-ECP calculations is demonstrated by comparing the respective supramolecular interaction energies $E_{\text {int,SAPT }}^{\text {DGDVP }}$ and $E_{\text {int,MP2 }}^{c c-p V T Z-E C P}$ (Figure FS7). Although deviations, for several systems even as large as 1.5 to $2.5 \mathrm{kcal} / \mathrm{mol}$, are clearly observed, we still note that both interaction energies reproduce each other on a qualitative basis. The analogy of the two methods is nicely 
seen in the second group (17 - 32) in which the electron-withdrawing cyano substituent replaced the methyl group in the accepting subunit. Here, the deviations between the two methods are purely quantitative and resemble closely the deviations observed for the interaction energies between MP2 and $\operatorname{CCSD}(\mathrm{T})$ (see Ref. 19 and also Figure FS1). Please note that a SAPT calculation is roughly equivalent to MBPT up to fourth order.

The deviations within the first group (model systems 1-16) are somewhat more elaborated, most notably the slope within the families $1-4,5-8,10-12$ and $13-16$ of the MP2/cc-pVTZ-ECP level is only marginally reproduced at the SAPT/DGDZVP level of theory. However, analogous deviations are noted for MP2/DGDZVP and $\operatorname{CCSD}(\mathrm{T}) / \mathrm{DGDZVP}$ levels of theory (see Figure FS1). It is noteworthy here, that the SAPT calculations are equivalent to highly correlated electronic structure calculations, and that the DGDZVP basis set employed in this work may not satisfy the demand in order to recover most of the correlation energy. The MP2/cc-pVTZ-ECP level of theory has been successfully correlated to the CCSD(T)/aug-cc-pVTZ-ECP interaction energy (see Ref. 19). However, throughout this work, the data obtained by the SAPT calculations are solely used in a qualitative manner to elucidate the nature (i.e. the composition) of the supramolecular interaction energy.

\section{S.3. Motivation for the partitioning of the SAPT interaction energy correction terms into electrostatic, induction, dispersion and exchange energy.}

The motivation for the partitioning of the interaction energy correction terms computed by the SAPT program comes from a comparison of the classical polarization expansion (PE) and the SAPT formalism. The difference between the SAPT and the PE formalism are mainly due to the quantum mechanically correct treatment of the Pauli principle for finite

intermonomeric distances by means of an anti-symmetrizing operator $\widehat{\mathbf{S}}^{(-)}$. While the PE 
calculates the interaction energy as (eq S4), the SAPT treatment includes the exchange energy originating from the Pauli principle (eq S5).

(eq S4) $E_{p o l, \text { int }}=\sum_{n=1}^{\infty} E_{p o l}^{(n)}$

(eq S5) $\quad E_{S A P T, \mathrm{int}}=\sum_{n=1}^{\infty} E_{S A P T}^{(n)}=\sum_{n=1}^{\infty}\left(E_{p o l}^{(n)}+E_{\text {exch }}^{(n)}\right)$

For practical reasons, the SAPT interaction energy is a double perturbation approach and each SAPT expansion coefficient $E_{S A P T}^{(n)}$ is therefore estimated as a (truncated) series using the Hartree-Fock wave function as the zeroth-order approximation. (Eq S5) therefore gives

$$
E_{S A P T, \text { int }}=\sum_{n=1}^{\infty} E_{S A P T}^{(n)}=\sum_{n=1}^{\infty} \sum_{k=0}^{\infty} E_{S A P T}^{(n k)}=\sum_{n=1}^{\infty} \sum_{k=0}^{\infty}\left(E_{p o l}^{(n k)}+E_{\text {exch }}^{(n k)}\right)
$$

The SAPT program used here computes $E_{S A P T, \text { int }}$ as follows:

$$
E_{S A P T, \text { int }}=E_{p o l}^{(10)}+E_{\text {exch }}^{(10)}+E_{\text {ind, resp }}^{(20)}+E_{\text {exch-ind, resp }}^{(20)}+\delta_{H F}+\varepsilon_{p o l}^{(1)}(3)+\varepsilon_{\text {exch }}^{(1)}(2)+E_{d i s p}^{(20)}+\varepsilon_{\text {disp }}^{(2)}(2)+E_{\text {exch-disp }}^{(20)},
$$

where $\mathcal{\varepsilon}^{(n)}(k)=\sum_{l=0}^{k} E^{(n k)}$.

Because the PE offers a rather simple and widely used classification of the different correction terms (electrostatic, induction and dispersion), we considered it useful to perform an analogous partitioning for the SAPT energy correction terms. Because all these 
terms from the PE expansion or represent their respective exchange terms (see eq S5), we found it appropriate to perform an energy partitioning into the electrostatic ( $E_{\text {elst }}$ ), induction $\left(E_{\text {ind }}\right)$, dispersion $\left(E_{\text {disp }}\right)$ and exchange $\left(E_{\text {exch }}\right)$ interaction energies. Towards this end, we have summed all SAPT correction terms originating from the PE's electrostatic terms plus their corresponding exchange terms into what we denote as electrostatic interaction $E_{\text {elst }}$ (eq S7). An analogous scheme was applied to yield the induction and dispersion interaction ( $E_{\text {ind }}$; eq $S 8$ and $E_{\text {disp }}$; eq $S 9$, respectively). The quantity denoted as $E_{\text {exch }}$ (eq S10) consists of those exchange energy correction terms for which no PE counterpart has been calculated by the SAPT program (i.e. it consists of only one correction term, see below).

(eq S7) $E_{\text {elst }}=E_{p o l}^{(10)}+E_{\text {exch }}^{(10)}+\varepsilon_{p o l}^{(1)}(3)$

(eq S8) $E_{\text {ind }}=E_{\text {ind, resp }}^{(20)}+E_{\text {exch-ind, resp }}^{(20)}$

(eq S9) $\quad E_{d i s p}=E_{d i s p}^{(20)}+\varepsilon_{d i s p}^{(2)}(2)+E_{\text {exch-disp }}^{(20)}$

(eq S10) $E_{\text {exch }}=\varepsilon_{\text {exch }}^{(1)}(2)$ 


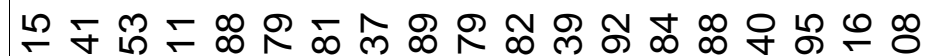
نִ

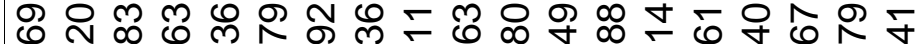

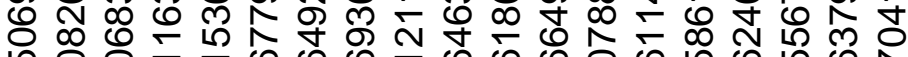

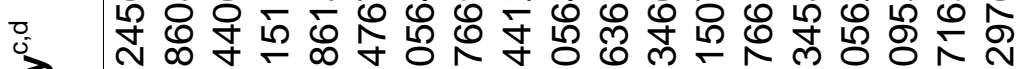
б ब

ง 는 ๒ N

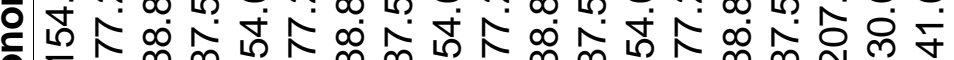
0

ம - đN N N N N क

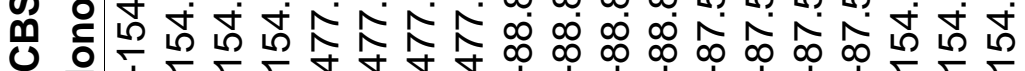

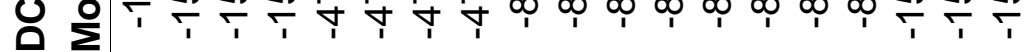

กั่ 它 ब

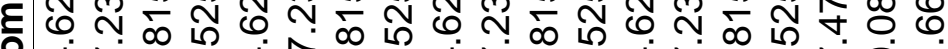

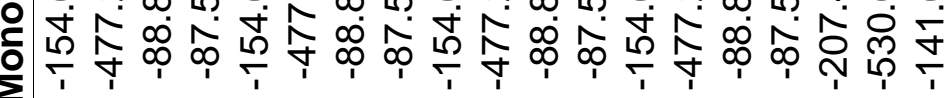

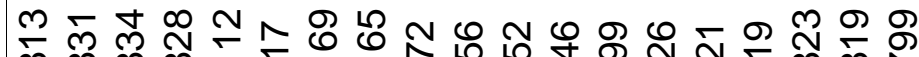

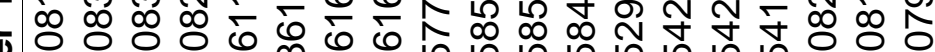

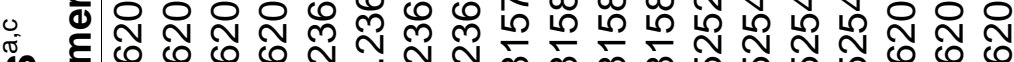

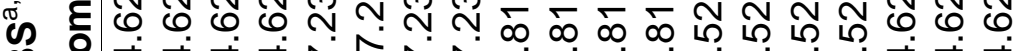

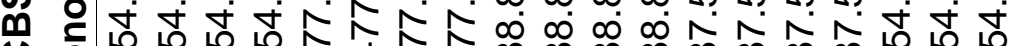
U。

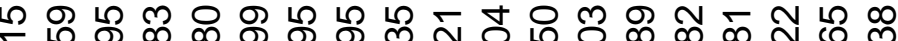
品 N $\bar{m}$ ल 鬲

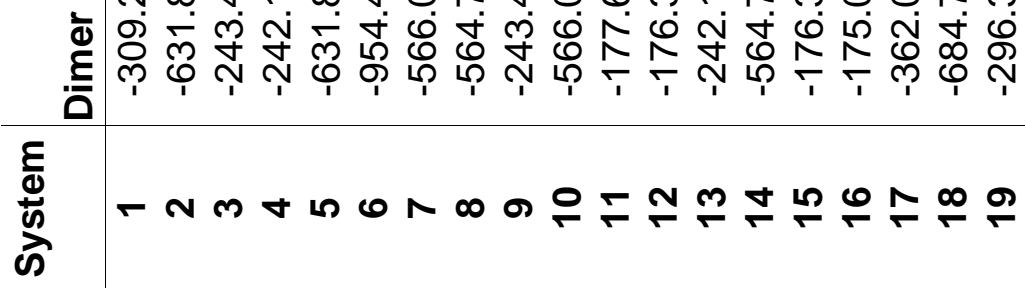




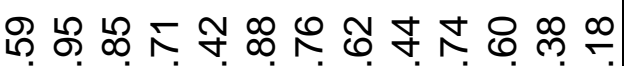
ம

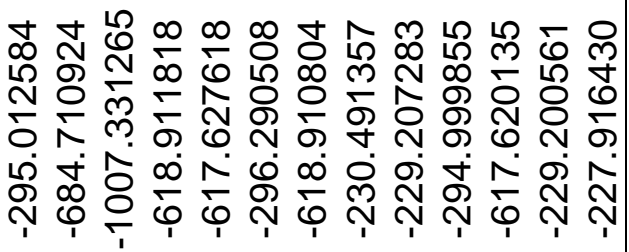

드 స్

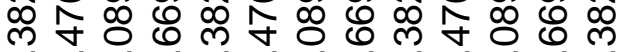
옹요

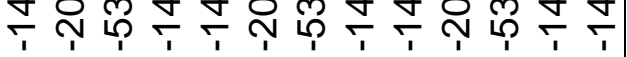

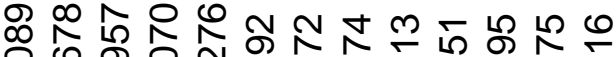

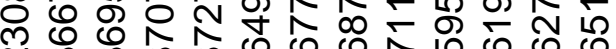
기 ल m O

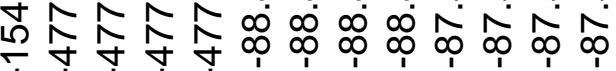

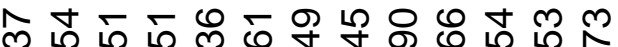
m 0 म N 0 क $\leftarrow \circ \infty$

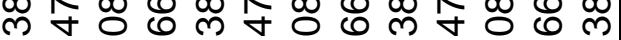

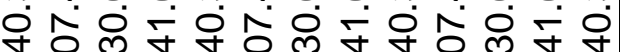

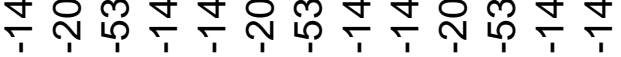

Ұ N

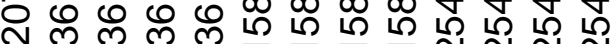

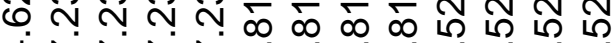

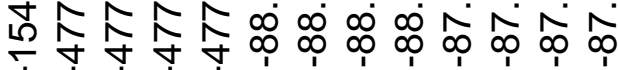

-

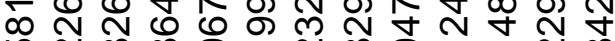
ద N m m क స б六 மं ํㅟ

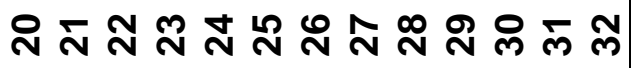

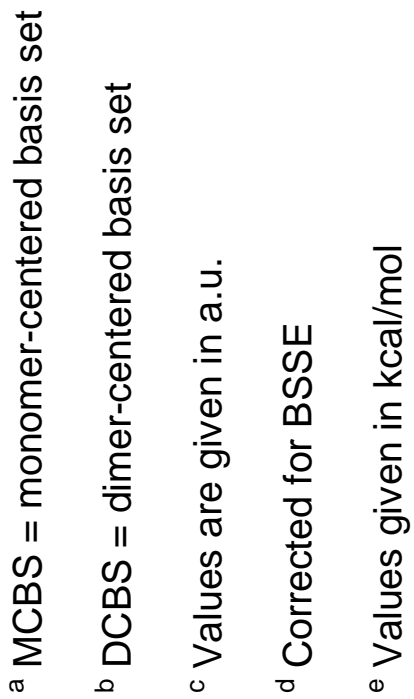


b) Cartesian coordinates of the optimized geometries for model systems 1-32 at the MP2/cc-pVTZ-ECP level of theory.

\begin{tabular}{lrrr}
$\begin{array}{l}\text { model } \\
\text { 18 }\end{array}$ & \multicolumn{3}{l}{ Stem 1 MS_J1.xyz } \\
& & & \\
$\mathrm{O}$ & -2.101743 & -0.000028 & 0.465098 \\
$\mathrm{O}$ & 1.510428 & 0.000015 & -0.257373 \\
$\mathrm{C}$ & -1.928812 & -1.166790 & -0.316714 \\
$\mathrm{C}$ & -1.928812 & 1.166828 & -0.316575 \\
$\mathrm{H}$ & -2.070561 & -2.019600 & 0.347364 \\
$\mathrm{H}$ & -2.671484 & -1.213932 & -1.125019 \\
$\mathrm{H}$ & -0.923456 & -1.203579 & -0.753562 \\
$\mathrm{H}$ & -2.070561 & 2.019559 & 0.347603 \\
$\mathrm{H}$ & -0.923456 & 1.203669 & -0.753419 \\
$\mathrm{H}$ & -2.671484 & 1.214065 & -1.124875 \\
$\mathrm{C}$ & 1.387814 & -0.000068 & 1.153273 \\
$\mathrm{C}$ & 2.864234 & 0.000039 & -0.658688 \\
$\mathrm{H}$ & 0.322936 & -0.000082 & 1.381943 \\
$\mathrm{H}$ & 1.857905 & -0.891805 & 1.589723 \\
$\mathrm{H}$ & 1.857905 & 0.891617 & 1.589829 \\
$\mathrm{H}$ & 2.877585 & 0.000104 & -1.748461 \\
$\mathrm{H}$ & 3.389324 & 0.891822 & -0.290299 \\
$\mathrm{H}$ & 3.389324 & -0.891788 & -0.290405
\end{tabular}

model system 2 last_o_s_Me_mp2_bsse.log.xyz 18

$\begin{array}{lrrr}\mathrm{O} & 2.192006 & 0.471231 & -0.003141 \\ \mathrm{~S} & -1.326477 & -0.394074 & 0.002407 \\ \mathrm{C} & 2.376815 & -0.312558 & -1.165847 \\ \mathrm{C} & 2.376717 & -0.296904 & 1.169981 \\ \mathrm{H} & 2.216177 & 0.342101 & -2.022265 \\ \mathrm{H} & 3.395514 & -0.721300 & -1.209291 \\ \mathrm{H} & 1.657892 & -1.140581 & -1.204332 \\ \mathrm{H} & 2.216019 & 0.369178 & 2.017535 \\ \mathrm{H} & 1.657782 & -1.124329 & 1.219506 \\ \mathrm{H} & 3.395409 & -0.705034 & 1.218981 \\ \mathrm{C} & -1.138309 & 1.404418 & -0.009189 \\ \mathrm{C} & -3.132928 & -0.491443 & 0.003372 \\ \mathrm{H} & -0.066290 & 1.603104 & -0.010680 \\ \mathrm{H} & -1.592342 & 1.832209 & -0.905644 \\ \mathrm{H} & -1.591982 & 1.843698 & 0.881875 \\ \mathrm{H} & -3.403262 & -1.548308 & 0.010217 \\ \mathrm{H} & -3.545382 & -0.012500 & 0.893847 \\ \mathrm{H} & -3.545717 & -0.023988 & -0.893033\end{array}$

$\begin{array}{lccc}\begin{array}{l}\text { model } \\ \text { 18 }\end{array} & \text { system } 3 & \text { last_o_Se_Me_mp2_bsse_freq.log.xyz } \\ \text { O } & 2.329200 & 0.449009 & -0.000041 \\ \mathrm{Se} & -0.961575 & -0.291869 & 0.000018 \\ \mathrm{C} & 2.686638 & -0.263745 & -1.167674 \\ \mathrm{C} & 2.686635 & -0.263539 & 1.167720 \\ \mathrm{H} & 2.374818 & 0.339624 & -2.019811 \\ \mathrm{H} & 3.771890 & -0.426958 & -1.215146 \\ \mathrm{H} & 2.178160 & -1.235464 & -1.210062 \\ \mathrm{H} & 2.374813 & 0.339981 & 2.019749 \\ & & & \mathrm{~S} 11\end{array}$




$\begin{array}{rrrr}\mathrm{H} & 2.178157 & -1.235249 & 1.210278 \\ \mathrm{H} & 3.771887 & -0.426743 & 1.215224 \\ \mathrm{C} & -0.858757 & 1.646780 & -0.000114 \\ \mathrm{C} & -2.903635 & -0.401971 & 0.000033 \\ \mathrm{H} & 0.203057 & 1.890779 & -0.000136 \\ \mathrm{H} & -1.335904 & 2.047947 & -0.895213 \\ \mathrm{H} & -1.335894 & 2.048070 & 0.894935 \\ \mathrm{H} & -3.168601 & -1.460164 & 0.000105 \\ \mathrm{H} & -3.308860 & 0.072313 & 0.894692 \\ \mathrm{H} & -3.308867 & 0.072192 & -0.894687\end{array}$

model system 4 last_O_Te_Me_mp2_bsse_freq.log.xyz 18

$\begin{array}{llcc}\mathrm{O} & 2.441585 & 0.394796 & -0.000708 \\ \mathrm{Te} & -0.780943 & -0.266225 & 0.000246 \\ \mathrm{C} & 2.946570 & -0.223432 & -1.168839 \\ \mathrm{C} & 2.946464 & -0.219246 & 1.169675 \\ \mathrm{H} & 2.509400 & 0.296005 & -2.020919 \\ \mathrm{H} & 4.040965 & -0.145633 & -1.215100 \\ \mathrm{H} & 2.663504 & -1.283285 & -1.209814 \\ \mathrm{H} & 2.509220 & 0.303242 & 2.019851 \\ \mathrm{H} & 2.663386 & -1.278943 & 1.214423 \\ \mathrm{H} & 4.040855 & -0.141283 & 1.215756 \\ \mathrm{C} & -0.611486 & 1.855699 & -0.002222 \\ \mathrm{C} & -2.916415 & -0.236849 & 0.000524 \\ \mathrm{H} & 0.454609 & 2.081041 & -0.002767 \\ \mathrm{H} & -1.080103 & 2.265609 & -0.896948 \\ \mathrm{H} & -1.079634 & 2.267691 & 0.891794 \\ \mathrm{H} & -3.267898 & -1.269852 & 0.001767 \\ \mathrm{H} & -3.284242 & 0.267886 & 0.893911 \\ \mathrm{H} & -3.284502 & 0.265821 & -0.893920\end{array}$

model system 5 last_S_O_Me_mp2_bsse_freq.log.xyz 18

$\begin{array}{lrrr}\mathrm{S} & 2.097905 & -0.000004 & 0.451236 \\ \mathrm{O} & -1.805633 & 0.000007 & -0.182561 \\ \mathrm{C} & 1.490953 & 1.370577 & -0.564095 \\ \mathrm{C} & 1.490948 & -1.370569 & -0.564115 \\ \mathrm{H} & 1.748047 & 2.296513 & -0.047321 \\ \mathrm{H} & 1.973757 & 1.364506 & -1.543665 \\ \mathrm{H} & 0.407938 & 1.302128 & -0.678506 \\ \mathrm{H} & 1.748038 & -2.296513 & -0.047355 \\ \mathrm{H} & 0.407933 & -1.302114 & -0.678525 \\ \mathrm{H} & 1.973752 & -1.364485 & -1.543685 \\ \mathrm{C} & -1.796477 & -0.000012 & 1.233987 \\ \mathrm{C} & -3.123654 & 0.000006 & -0.691899 \\ \mathrm{H} & -0.752629 & -0.000008 & 1.546340 \\ \mathrm{H} & -2.299093 & 0.891977 & 1.631376 \\ \mathrm{H} & -2.299079 & -0.892018 & 1.631353 \\ \mathrm{H} & -3.048963 & 0.000019 & -1.779153 \\ \mathrm{H} & -3.675858 & -0.891911 & -0.366713 \\ \mathrm{H} & -3.675868 & 0.891909 & -0.366692\end{array}$

model system 6 MS_J4.xyz

18

$\begin{array}{lrrr}\text { S } & -2.280942 & 0.548472 & -0.000612 \\ \text { S } & 1.653807 & -0.332347 & 0.000249 \\ \text { C } & -2.051599 & -0.604565 & 1.375349 \\ \text { C } & -2.051683 & -0.607733 & -1.373926\end{array}$

$\mathrm{S} 12$ 


$\begin{array}{rrrr}\mathrm{H} & -2.170646 & -0.034339 & 2.297862 \\ \mathrm{H} & -2.807404 & -1.392155 & 1.343976 \\ \mathrm{H} & -1.050992 & -1.039292 & 1.349106 \\ \mathrm{H} & -2.170771 & -0.039630 & -2.297743 \\ \mathrm{H} & -1.051080 & -1.042409 & -1.346736 \\ \mathrm{H} & -2.807494 & -1.395240 & -1.340700 \\ \mathrm{C} & 1.634518 & 1.476038 & -0.001604 \\ \mathrm{C} & 3.443151 & -0.599182 & 0.000744 \\ \mathrm{H} & 0.586581 & 1.777628 & -0.002036 \\ \mathrm{H} & 2.125879 & 1.866098 & 0.892227 \\ \mathrm{H} & 2.126094 & 1.864269 & -0.896111 \\ \mathrm{H} & 3.612407 & -1.676817 & 0.001860 \\ \mathrm{H} & 3.897745 & -0.167643 & -0.893313 \\ \mathrm{H} & 3.897523 & -0.165827 & 0.894036\end{array}$

\section{model system 7 last_S_Se_Me_mp2_bsse_001.log.xyz 18}

$\begin{array}{llcc}\mathrm{S} & 2.461871 & 0.572040 & -0.149642 \\ \mathrm{Se} & -1.228969 & -0.241668 & 0.022307 \\ \mathrm{C} & 2.458435 & -0.919071 & -1.173615 \\ \mathrm{C} & 2.450420 & -0.210399 & 1.481336 \\ \mathrm{H} & 2.466179 & -0.596104 & -2.215595 \\ \mathrm{H} & 3.349332 & -1.519766 & -0.978875 \\ \mathrm{H} & 1.557445 & -1.507213 & -0.990450 \\ \mathrm{H} & 2.460037 & 0.588951 & 2.223743 \\ \mathrm{H} & 1.546020 & -0.807136 & 1.612371 \\ \mathrm{H} & 3.338403 & -0.831872 & 1.614512 \\ \mathrm{C} & -1.382807 & 1.665112 & -0.312699 \\ \mathrm{C} & -3.138803 & -0.585979 & 0.159894 \\ \mathrm{H} & -0.365301 & 2.040096 & -0.421056 \\ \mathrm{H} & -1.943399 & 1.839620 & -1.231838 \\ \mathrm{H} & -1.872228 & 2.155339 & 0.529749 \\ \mathrm{H} & -3.262328 & -1.651913 & 0.355720 \\ \mathrm{H} & -3.566459 & -0.014226 & 0.984278 \\ \mathrm{H} & -3.636180 & -0.329670 & -0.776222\end{array}$

model system 8 last_S_Te_Me_mp2_bsse.log.xyz 18

$\begin{array}{llrr}\mathrm{S} & 2.579926 & 0.452894 & -0.390928 \\ \mathrm{Te} & -0.999411 & -0.233885 & 0.017921 \\ \mathrm{C} & 2.734467 & -1.329727 & -0.651748 \\ \mathrm{C} & 2.783969 & 0.488907 & 1.406135 \\ \mathrm{H} & 2.606339 & -1.513601 & -1.719195 \\ \mathrm{H} & 3.721721 & -1.678331 & -0.341958 \\ \mathrm{H} & 1.957450 & -1.865801 & -0.102743 \\ \mathrm{H} & 2.719868 & 1.532127 & 1.718862 \\ \mathrm{H} & 1.986675 & -0.078171 & 1.890405 \\ \mathrm{H} & 3.760319 & 0.090345 & 1.689328 \\ \mathrm{C} & -1.131883 & 1.793849 & -0.619165 \\ \mathrm{C} & -3.099905 & -0.395438 & 0.374505 \\ \mathrm{H} & -0.124523 & 2.109744 & -0.890052 \\ \mathrm{H} & -1.786385 & 1.865070 & -1.487835 \\ \mathrm{H} & -1.510042 & 2.415648 & 0.192236 \\ \mathrm{H} & -3.303786 & -1.406051 & 0.731693 \\ \mathrm{H} & -3.406969 & 0.322989 & 1.134561 \\ \mathrm{H} & -3.650029 & -0.223816 & -0.550726\end{array}$

model system 9 last_Se_O_Me_mp2_bsse_freq.log.xyz 18 


$\begin{array}{lrrr}\mathrm{Se} & 1.696925 & -0.000002 & 0.281757 \\ \mathrm{O} & -2.336441 & 0.000008 & -0.159519 \\ \mathrm{C} & 0.906994 & 1.442220 & -0.757323 \\ \mathrm{C} & 0.906992 & -1.442211 & -0.757339 \\ \mathrm{H} & 1.186911 & 2.379452 & -0.274261 \\ \mathrm{H} & 1.299058 & 1.427248 & -1.774911 \\ \mathrm{H} & -0.177539 & 1.332524 & -0.762245 \\ \mathrm{H} & 1.186906 & -2.379449 & -0.274287 \\ \mathrm{H} & -0.177541 & -1.332513 & -0.762261 \\ \mathrm{H} & 1.299057 & -1.427230 & -1.774927 \\ \mathrm{C} & -2.277124 & -0.000009 & 1.255722 \\ \mathrm{C} & -3.671650 & 0.000002 & -0.622100 \\ \mathrm{H} & -1.222740 & -0.000004 & 1.530429 \\ \mathrm{H} & -2.765044 & 0.892060 & 1.670852 \\ \mathrm{H} & -2.765029 & -0.892097 & 1.670830 \\ \mathrm{H} & -3.635237 & 0.000016 & -1.711302 \\ \mathrm{H} & -4.211994 & -0.891938 & -0.277651 \\ \mathrm{H} & -4.212010 & 0.891925 & -0.277628\end{array}$

\section{model system 10 last_Se_S_Me_mp2_bsse.log.xyz 18}

$\begin{array}{llrr}\mathrm{Se} & -1.905685 & 0.366103 & -0.000704 \\ \mathrm{~S} & 2.199372 & -0.329296 & 0.000339 \\ \mathrm{C} & -1.480893 & -0.857499 & 1.449834 \\ \mathrm{C} & -1.481097 & -0.863262 & -1.446421 \\ \mathrm{H} & -1.672802 & -0.326264 & 2.383110 \\ \mathrm{H} & -2.124068 & -1.736514 & 1.394248 \\ \mathrm{H} & -0.430339 & -1.143992 & 1.400712 \\ \mathrm{H} & -1.673136 & -0.335744 & -2.381776 \\ \mathrm{H} & -0.430536 & -1.149559 & -1.396310 \\ \mathrm{H} & -2.124264 & -1.742049 & -1.387246 \\ \mathrm{C} & 2.096381 & 1.476309 & -0.002544 \\ \mathrm{C} & 3.999119 & -0.513338 & 0.001044 \\ \mathrm{H} & 1.035526 & 1.729087 & -0.003174 \\ \mathrm{H} & 2.568799 & 1.889341 & 0.891174 \\ \mathrm{H} & 2.569191 & 1.886509 & -0.897358 \\ \mathrm{H} & 4.217742 & -1.582041 & 0.002778 \\ \mathrm{H} & 4.433284 & -0.061808 & -0.893281 \\ \mathrm{H} & 4.432873 & -0.058990 & 0.894141\end{array}$

model system 11 MS_J7.xyz

18

$\begin{array}{llrr}\mathrm{Se} & 2.144337 & 0.407318 & -0.043948 \\ \mathrm{Se} & -1.706192 & -0.241890 & 0.003082 \\ \mathrm{C} & 1.953806 & -1.026943 & -1.341859 \\ \mathrm{C} & 1.948118 & -0.708636 & 1.535429 \\ \mathrm{H} & 2.051349 & -0.578626 & -2.331479 \\ \mathrm{H} & 2.742600 & -1.766212 & -1.197998 \\ \mathrm{H} & 0.969807 & -1.486264 & -1.246609 \\ \mathrm{H} & 2.045995 & -0.055383 & 2.403530 \\ \mathrm{H} & 0.962736 & -1.174793 & 1.540397 \\ \mathrm{H} & 2.735063 & -1.463383 & 1.557902 \\ \mathrm{C} & -1.789279 & 1.695017 & -0.113395 \\ \mathrm{C} & -3.629143 & -0.531933 & 0.061393 \\ \mathrm{H} & -0.757565 & 2.043505 & -0.156557 \\ \mathrm{H} & -2.320671 & 1.993331 & -1.017915 \\ \mathrm{H} & -2.282793 & 2.102677 & 0.769671 \\ \mathrm{H} & -3.792098 & -1.608249 & 0.130563 \\ \mathrm{H} & -4.057403 & -0.043514 & 0.937416 \\ \mathrm{H} & -4.094960 & -0.152686 & -0.848860\end{array}$

$\mathrm{S} 14$ 


$\begin{array}{lccc}\text { model } & \text { system } 12 & \text { last_Se_Te_Me_mp2_bsse_freq.log } \\ & & & \\ \text { Se } & 2.303356 & 0.318076 & -0.288534 \\ \mathrm{Te} & -1.419392 & -0.239602 & -0.004233 \\ \mathrm{C} & 2.274404 & -1.614038 & -0.489954 \\ \mathrm{C} & 2.350067 & 0.323483 & 1.654639 \\ \mathrm{H} & 2.211921 & -1.825262 & -1.558059 \\ \mathrm{H} & 3.189798 & -2.045062 & -0.083274 \\ \mathrm{H} & 1.399233 & -2.026468 & 0.013687 \\ \mathrm{H} & 2.382451 & 1.366371 & 1.972902 \\ \mathrm{H} & 1.448185 & -0.149422 & 2.044493 \\ \mathrm{H} & 3.243550 & -0.192519 & 2.007830 \\ \mathrm{C} & -1.483030 & 1.834032 & -0.486238 \\ \mathrm{C} & -3.525014 & -0.353326 & 0.343043 \\ \mathrm{H} & -0.465367 & 2.135726 & -0.733833 \\ \mathrm{H} & -2.133728 & 1.992523 & -1.346190 \\ \mathrm{H} & -1.840797 & 2.405104 & 0.370447 \\ \mathrm{H} & -3.764508 & -1.380074 & 0.623952 \\ \mathrm{H} & -3.807209 & 0.316619 & 1.155245 \\ \mathrm{H} & -4.067815 & -0.093745 & -0.565884\end{array}$

\section{model system 13 last_Te_O_Me_mp2_bsse_freq.log.xyz} 18

$\begin{array}{lrrr}\mathrm{Te} & -1.460151 & -0.000003 & -0.201910 \\ \mathrm{O} & 2.705807 & 0.000013 & 0.107002 \\ \mathrm{C} & -0.455964 & 1.552897 & 0.860698 \\ \mathrm{C} & -0.455956 & -1.552880 & 0.860724 \\ \mathrm{H} & -0.726129 & 2.504834 & 0.401249 \\ \mathrm{H} & -0.769203 & 1.551834 & 1.904397 \\ \mathrm{H} & 0.618824 & 1.391637 & 0.781153 \\ \mathrm{H} & -0.726115 & -2.504825 & 0.401290 \\ \mathrm{H} & 0.618832 & -1.391614 & 0.781176 \\ \mathrm{H} & -0.769196 & -1.551802 & 1.904423 \\ \mathrm{C} & 2.701684 & -0.000014 & -1.309232 \\ \mathrm{C} & 4.022214 & 0.000003 & 0.621266 \\ \mathrm{H} & 1.658803 & -0.000003 & -1.624804 \\ \mathrm{H} & 3.204840 & 0.892164 & -1.705399 \\ \mathrm{H} & 3.204810 & -0.892223 & -1.705368 \\ \mathrm{H} & 3.943225 & 0.000024 & 1.708207 \\ \mathrm{H} & 4.575402 & -0.891993 & 0.298146 \\ \mathrm{H} & 4.575428 & 0.891971 & 0.298113\end{array}$

model system 14 last_Te_S_Me_mp2_bsse_freq.log.xyz 18

$\begin{array}{lrrr}\text { Te } & -1.640439 & 0.285462 & -0.000321 \\ \mathrm{~S} & 2.545157 & -0.274716 & 0.000078 \\ \mathrm{C} & -1.051841 & -1.040786 & 1.562078 \\ \mathrm{C} & -1.052003 & -1.044479 & -1.559638 \\ \mathrm{H} & -1.283050 & -0.557754 & 2.512660 \\ \mathrm{H} & -1.613746 & -1.971395 & 1.483885 \\ \mathrm{H} & 0.019371 & -1.228240 & 1.497773 \\ \mathrm{H} & -1.283326 & -0.563705 & -2.511336 \\ \mathrm{H} & 0.019217 & -1.231772 & -1.495015 \\ \mathrm{H} & -1.613890 & -1.974906 & -1.479175 \\ \mathrm{C} & 2.550210 & 1.533775 & -0.001490 \\ \mathrm{C} & 4.331400 & -0.563229 & 0.000665 \\ \mathrm{H} & 1.506729 & 1.850323 & -0.001959 \\ \mathrm{H} & 3.046254 & 1.917265 & 0.892582\end{array}$




$\begin{array}{rrrr}\mathrm{H} & 3.046591 & 1.915709 & -0.896041 \\ \mathrm{H} & 4.487270 & -1.642878 & 0.001627 \\ \mathrm{H} & 4.791311 & -0.137232 & -0.893333 \\ \mathrm{H} & 4.790975 & -0.135689 & 0.894100\end{array}$

model system 15 last_Te_Se_Me_mp2_bsse_freq.log.xyz 18

$\begin{array}{lccr}\mathrm{Te} & 1.949357 & 0.315530 & -0.000234 \\ \mathrm{Se} & -2.100024 & -0.218140 & -0.000020 \\ \mathrm{C} & 1.523736 & -1.073971 & -1.559855 \\ \mathrm{C} & 1.523656 & -1.071538 & 1.561530 \\ \mathrm{H} & 1.711937 & -0.573779 & -2.511001 \\ \mathrm{H} & 2.180956 & -1.938782 & -1.469908 \\ \mathrm{H} & 0.477630 & -1.373717 & -1.507470 \\ \mathrm{H} & 1.711822 & -0.569869 & 2.511905 \\ \mathrm{H} & 0.477549 & -1.371353 & 1.509560 \\ \mathrm{H} & 2.180873 & -1.936495 & 1.472958 \\ \mathrm{C} & -2.193319 & 1.722090 & -0.000799 \\ \mathrm{C} & -4.021873 & -0.521566 & 0.000453 \\ \mathrm{H} & -1.163658 & 2.079626 & -0.001133 \\ \mathrm{H} & -2.707904 & 2.071631 & -0.896627 \\ \mathrm{H} & -2.707578 & 2.072367 & 0.894930 \\ \mathrm{H} & -4.178712 & -1.600978 & 0.000922 \\ \mathrm{H} & -4.470750 & -0.089418 & 0.895478 \\ \mathrm{H} & -4.471074 & -0.090149 & -0.894762\end{array}$

model system 16 MS_J10.xyz 18

$\begin{array}{lrrr}\mathrm{Te} & -2.144913 & 0.259446 & -0.220702 \\ \mathrm{Te} & 1.792567 & -0.228219 & -0.027026 \\ \mathrm{C} & -1.971452 & 0.165636 & 1.901597 \\ \mathrm{C} & -1.888868 & -1.839927 & -0.479129 \\ \mathrm{H} & -2.087466 & 1.177823 & 2.291887 \\ \mathrm{H} & -2.756508 & -0.471550 & 2.308382 \\ \mathrm{H} & -0.985055 & -0.216123 & 2.164399 \\ \mathrm{H} & -1.900995 & -2.048959 & -1.549627 \\ \mathrm{H} & -0.928812 & -2.143790 & -0.061738 \\ \mathrm{H} & -2.705203 & -2.373979 & 0.006583 \\ \mathrm{C} & 1.860736 & 1.862659 & -0.428517 \\ \mathrm{C} & 3.894188 & -0.355681 & 0.340196 \\ \mathrm{H} & 0.845193 & 2.174896 & -0.672013 \\ \mathrm{H} & 2.211802 & 2.399500 & 0.452710 \\ \mathrm{H} & 2.518360 & 2.053357 & -1.276558 \\ \mathrm{H} & 4.130105 & -1.392438 & 0.584859 \\ \mathrm{H} & 4.446850 & -0.061982 & -0.552217 \\ \mathrm{H} & 4.166106 & 0.283282 & 1.180346\end{array}$

model system 17 MS_J3.xyz 16

$\begin{array}{rrrr}\mathrm{O} & 2.197311 & 0.393260 & -0.000001 \\ \mathrm{O} & -1.199026 & -0.049611 & 0.000000 \\ \mathrm{C} & 2.242974 & -0.404428 & -1.168369 \\ \mathrm{C} & 2.242974 & -0.404424 & 1.168371 \\ \mathrm{H} & 2.212403 & 0.273620 & -2.021129 \\ \mathrm{H} & 3.169271 & -0.992072 & -1.209221 \\ \mathrm{H} & 1.385988 & -1.088343 & -1.214587 \\ \mathrm{H} & 2.212403 & 0.273627 & 2.021128 \\ \mathrm{H} & 1.385988 & -1.088339 & 1.214591 \\ \mathrm{H} & 3.169271 & -0.992068 & 1.209225\end{array}$

$\mathrm{S} 16$ 


$\begin{array}{rrrr}\mathrm{C} & -2.442941 & -0.408386 & 0.000001 \\ \mathrm{C} & -0.999361 & 1.395437 & -0.000002 \\ \mathrm{H} & 0.079551 & 1.519392 & -0.000003 \\ \mathrm{H} & -1.450726 & 1.820869 & 0.896700 \\ \mathrm{H} & -1.450727 & 1.820866 & -0.896706 \\ \mathrm{~N} & -3.565939 & -0.766562 & 0.000001\end{array}$

\section{model system 18 last_o_s_CN_mp2_bsse_freq.log.xyz 16}

$\begin{array}{rrrr}\mathrm{O} & 2.129220 & 0.314421 & -0.000007 \\ \mathrm{~S} & -0.906429 & -0.229474 & 0.000000 \\ \mathrm{C} & 2.576597 & -0.346162 & -1.169787 \\ \mathrm{C} & 2.576589 & -0.346113 & 1.169804 \\ \mathrm{H} & 2.194545 & 0.215049 & -2.022120 \\ \mathrm{H} & 3.672971 & -0.371243 & -1.213368 \\ \mathrm{H} & 2.195124 & -1.374348 & -1.213517 \\ \mathrm{H} & 2.194530 & 0.215134 & 2.022110 \\ \mathrm{H} & 2.195115 & -1.374297 & 1.213574 \\ \mathrm{H} & 3.672963 & -0.371191 & 1.213394 \\ \mathrm{C} & -2.590901 & -0.442201 & 0.000007 \\ \mathrm{C} & -0.867900 & 1.592390 & -0.000027 \\ \mathrm{H} & 0.192591 & 1.841211 & -0.000034 \\ \mathrm{H} & -1.352788 & 1.974859 & 0.896608 \\ \mathrm{H} & -1.352793 & 1.974831 & -0.896672 \\ \mathrm{~N} & -3.758496 & -0.617324 & 0.000012\end{array}$

\section{model system 19 last_O_Se_CN_mp2_bsse_freq.log.xyz 16}

$\begin{array}{lccc}\mathrm{O} & -2.265566 & 0.278590 & -0.000005 \\ \mathrm{Se} & 0.681952 & -0.222402 & 0.000002 \\ \mathrm{C} & -2.832244 & -0.282810 & 1.171417 \\ \mathrm{C} & -2.832245 & -0.282851 & -1.171407 \\ \mathrm{H} & -2.348039 & 0.194335 & 2.022834 \\ \mathrm{H} & -3.912433 & -0.095123 & 1.213688 \\ \mathrm{H} & -2.656962 & -1.365283 & 1.215003 \\ \mathrm{H} & -2.348041 & 0.194264 & -2.022841 \\ \mathrm{H} & -2.656962 & -1.365326 & -1.214955 \\ \mathrm{H} & -3.912433 & -0.095167 & -1.213685 \\ \mathrm{C} & 2.524024 & -0.351586 & 0.000005 \\ \mathrm{C} & 0.643937 & 1.728704 & -0.000023 \\ \mathrm{H} & -0.415934 & 1.979433 & -0.000028 \\ \mathrm{H} & 1.133115 & 2.101246 & -0.897650 \\ \mathrm{H} & 1.133112 & 2.101270 & 0.897595 \\ \mathrm{~N} & 3.700270 & -0.455060 & 0.000008\end{array}$

\section{model system 20 last_O_Te_CN_mp2_bsse.2_freq.log.xyz} 16

$\begin{array}{llrr}\mathrm{O} & 2.360152 & 0.241233 & -0.000005 \\ \mathrm{Te} & -0.541923 & -0.238916 & 0.000000 \\ \mathrm{C} & 3.020168 & -0.207885 & -1.174061 \\ \mathrm{C} & 3.020159 & -0.207825 & 1.174078 \\ \mathrm{H} & 2.458404 & 0.176346 & -2.024529 \\ \mathrm{H} & 4.047983 & 0.172510 & -1.211436 \\ \mathrm{H} & 3.042418 & -1.303820 & -1.218128 \\ \mathrm{H} & 2.458387 & 0.176448 & 2.024523 \\ \mathrm{H} & 3.042410 & -1.303758 & 1.218200 \\ \mathrm{H} & 4.047973 & 0.172574 & 1.211442 \\ \mathrm{C} & -2.593316 & -0.235190 & 0.000008 \\ \mathrm{C} & -0.460677 & 1.890818 & -0.000033\end{array}$




$\begin{array}{rrrr}\mathrm{H} & 0.599012 & 2.143738 & -0.000039 \\ \mathrm{H} & -0.947372 & 2.271306 & 0.895778 \\ \mathrm{H} & -0.947374 & 2.271278 & -0.895855 \\ \mathrm{~N} & -3.774439 & -0.246054 & 0.000012\end{array}$

model system 21 last_s_o_CN_mp2_bsse.log.xyz 16

$\begin{array}{lrrr}\mathrm{S} & -2.260887 & 0.426928 & -0.000023 \\ \mathrm{O} & 1.521922 & 0.048070 & -0.000007 \\ \mathrm{C} & -1.809496 & -0.663203 & 1.373620 \\ \mathrm{C} & -1.809492 & -0.663345 & -1.373552 \\ \mathrm{H} & -2.022694 & -0.125613 & 2.298800 \\ \mathrm{H} & -2.406589 & -1.576977 & 1.348283 \\ \mathrm{H} & -0.746901 & -0.909569 & 1.333856 \\ \mathrm{H} & -2.022687 & -0.125851 & -2.298789 \\ \mathrm{H} & -0.746898 & -0.909708 & -1.333760 \\ \mathrm{H} & -2.406586 & -1.577116 & -1.348123 \\ \mathrm{C} & 2.732457 & -0.415160 & 0.000019 \\ \mathrm{C} & 1.446986 & 1.505882 & -0.000069 \\ \mathrm{H} & 0.382580 & 1.724287 & -0.000083 \\ \mathrm{H} & 1.931730 & 1.890920 & -0.897488 \\ \mathrm{H} & 1.931720 & 1.890997 & 0.897322 \\ \mathrm{~N} & 3.820343 & -0.868831 & 0.000043\end{array}$

model system 22 MS_J6.xyz 16

$\begin{array}{lrrr}\mathrm{S} & -2.234755 & 0.505344 & -0.000969 \\ \mathrm{~S} & 1.205103 & -0.099941 & -0.000287 \\ \mathrm{C} & -2.289699 & -0.666869 & 1.375791 \\ \mathrm{C} & -2.289763 & -0.672013 & -1.373328 \\ \mathrm{H} & -2.264037 & -0.085950 & 2.298701 \\ \mathrm{H} & -3.213592 & -1.247536 & 1.345129 \\ \mathrm{H} & -1.425573 & -1.333551 & 1.348732 \\ \mathrm{H} & -2.264079 & -0.094556 & -2.298406 \\ \mathrm{H} & -1.425670 & -1.338632 & -1.343783 \\ \mathrm{H} & -3.213681 & -1.252522 & -1.340481 \\ \mathrm{C} & 2.843012 & -0.547962 & 0.000747 \\ \mathrm{C} & 1.425579 & 1.709151 & -0.002058 \\ \mathrm{H} & 0.413155 & 2.111824 & -0.002850 \\ \mathrm{H} & 1.959088 & 2.017742 & -0.899469 \\ \mathrm{H} & 1.958416 & 2.019584 & 0.895117 \\ \mathrm{~N} & 3.973661 & -0.888099 & 0.001498\end{array}$

model system 23 last_S_Se_CN_mp2_bsse_freq.log.xyz 16

$\begin{array}{llcc}\mathrm{S} & 2.400001 & 0.516390 & -0.000062 \\ \mathrm{Se} & -0.917798 & -0.113295 & -0.000002 \\ \mathrm{C} & 2.597211 & -0.641958 & -1.375571 \\ \mathrm{C} & 2.597222 & -0.641642 & 1.375712 \\ \mathrm{H} & 2.496372 & -0.070050 & -2.298827 \\ \mathrm{H} & 3.586106 & -1.103094 & -1.345742 \\ \mathrm{H} & 1.822742 & -1.411119 & -1.345742 \\ \mathrm{H} & 2.496392 & -0.069523 & 2.298838 \\ \mathrm{H} & 1.822752 & -1.410809 & 1.346067 \\ \mathrm{H} & 3.586116 & -1.102786 & 1.345980 \\ \mathrm{C} & -2.718322 & -0.529702 & 0.000047 \\ \mathrm{C} & -1.193262 & 1.819322 & -0.000128 \\ \mathrm{H} & -0.190513 & 2.245162 & -0.000169 \\ \mathrm{H} & -1.735374 & 2.107274 & 0.898125\end{array}$

S18 


$\begin{array}{rrrr}\mathrm{H} & -1.735397 & 2.107154 & -0.898405 \\ \mathrm{~N} & -3.863022 & -0.819792 & 0.000082\end{array}$

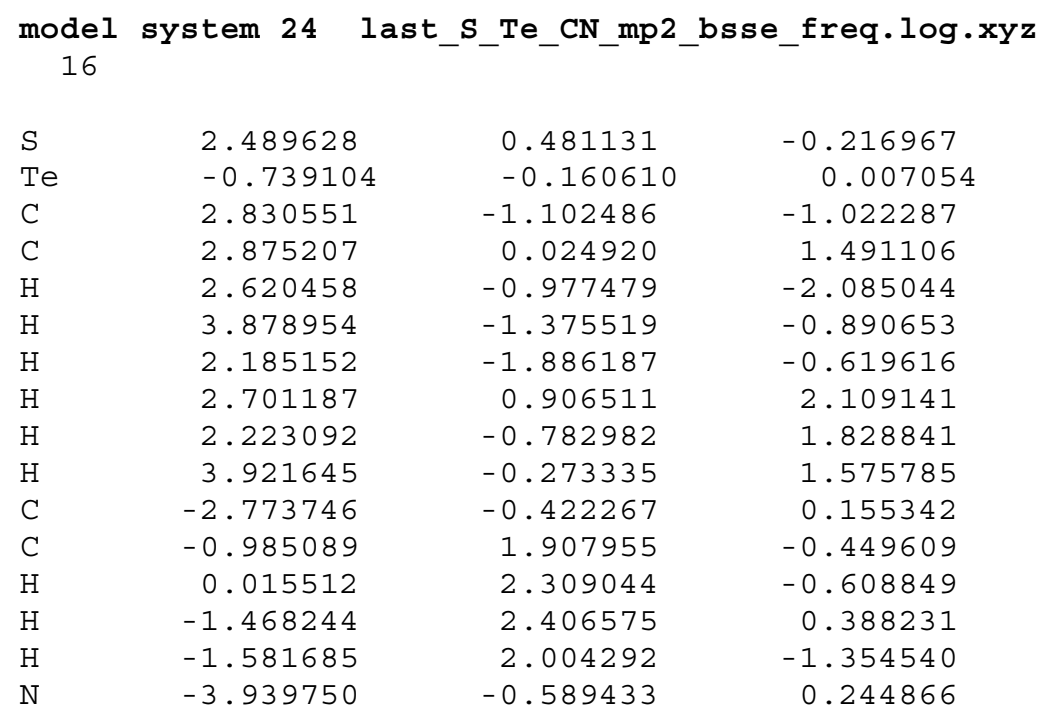

model system 25 last_Se_O_CN_mp2_bsse_freq.log.xyz 16

$\begin{array}{llcr}\mathrm{Se} & -1.859935 & 0.277898 & -0.000002 \\ \mathrm{O} & 2.073228 & 0.043464 & 0.000013 \\ \mathrm{C} & -1.220508 & -0.855908 & 1.445070 \\ \mathrm{C} & -1.220495 & -0.855909 & -1.445068 \\ \mathrm{H} & -1.476948 & -0.360256 & 2.382253 \\ \mathrm{H} & -1.714685 & -1.826839 & 1.401696 \\ \mathrm{H} & -0.139239 & -0.974748 & 1.373550 \\ \mathrm{H} & -1.476923 & -0.360255 & -2.382254 \\ \mathrm{H} & -0.139227 & -0.974751 & -1.373536 \\ \mathrm{H} & -1.714675 & -1.826839 & -1.401701 \\ \mathrm{C} & 3.301154 & -0.369464 & 0.000003 \\ \mathrm{C} & 1.937562 & 1.496015 & 0.000000 \\ \mathrm{H} & 0.864990 & 1.669707 & 0.000011 \\ \mathrm{H} & 2.405313 & 1.901621 & -0.897354 \\ \mathrm{H} & 2.405336 & 1.901640 & 0.897333 \\ \mathrm{~N} & 4.407391 & -0.776276 & -0.000006\end{array}$

\begin{tabular}{lccc} 
model & system 26 & last_Se_S_CN_mp2_bsse_freq.log.xyz \\
& \multicolumn{4}{c}{. } \\
Se & 1.881248 & 0.363077 & -0.000175 \\
$\mathrm{~S}$ & -1.700389 & -0.108795 & -0.000091 \\
$\mathrm{C}$ & 1.765481 & -0.929831 & -1.446834 \\
$\mathrm{C}$ & 1.765465 & -0.928490 & 1.447681 \\
$\mathrm{H}$ & 1.818569 & -0.371956 & -2.382521 \\
$\mathrm{H}$ & 2.603513 & -1.624991 & -1.389740 \\
$\mathrm{H}$ & 0.817323 & -1.465813 & -1.395084 \\
$\mathrm{H}$ & 1.818566 & -0.369751 & 2.382851 \\
$\mathrm{H}$ & 0.817298 & -1.464504 & 1.396428 \\
$\mathrm{H}$ & 2.603487 & -1.623715 & 1.391228 \\
$\mathrm{C}$ & -3.348867 & -0.516409 & 0.000148 \\
$\mathrm{C}$ & -1.876408 & 1.705121 & -0.000419 \\
$\mathrm{H}$ & -0.854047 & 2.081989 & -0.000598 \\
$\mathrm{H}$ & -2.401313 & 2.028048 & 0.897030 \\
$\mathrm{H}$ & -2.401502 & 2.027703 & -0.897881 \\
$\mathrm{~N}$ & -4.487449 & -0.829037 & 0.000321
\end{tabular}




\begin{tabular}{lrrr} 
model & system 27 & MS_J9.xyz \\
& \multicolumn{4}{l}{ 16 } & & & \\
$\mathrm{Se}$ & 2.101406 & 0.361067 & -0.123155 \\
$\mathrm{Se}$ & -1.359477 & -0.127141 & -0.020369 \\
$\mathrm{C}$ & 2.103976 & -1.314479 & -1.107697 \\
$\mathrm{C}$ & 2.155601 & -0.433082 & 1.650189 \\
$\mathrm{H}$ & 2.061784 & -1.064567 & -2.168353 \\
$\mathrm{H}$ & 3.021110 & -1.865225 & -0.897612 \\
$\mathrm{H}$ & 1.229944 & -1.909072 & -0.839045 \\
$\mathrm{H}$ & 2.165030 & 0.386410 & 2.369757 \\
$\mathrm{H}$ & 1.270242 & -1.049172 & 1.810861 \\
$\mathrm{H}$ & 3.063227 & -1.026731 & 1.761822 \\
$\mathrm{C}$ & -3.167802 & -0.486685 & 0.109363 \\
$\mathrm{C}$ & -1.589589 & 1.796968 & -0.256917 \\
$\mathrm{H}$ & -0.578320 & 2.188228 & -0.362516 \\
$\mathrm{H}$ & -2.076152 & 2.214626 & 0.622008 \\
$\mathrm{H}$ & -2.171265 & 1.982947 & -1.157320 \\
$\mathrm{~N}$ & -4.317758 & -0.741038 & 0.195799
\end{tabular}

$\underset{16}{\operatorname{model} \text { system } 28 \text { last_Se_Te_CN_mp2_bsse_freq.log.xyz }}$

$\begin{array}{lrrr}\mathrm{Se} & 2.234184 & 0.314519 & -0.251254 \\ \mathrm{Te} & -1.129555 & -0.179985 & -0.015130 \\ \mathrm{C} & 2.373314 & -1.586561 & -0.627956 \\ \mathrm{C} & 2.499933 & 0.174914 & 1.669472 \\ \mathrm{H} & 2.214015 & -1.714627 & -1.698977 \\ \mathrm{H} & 3.367255 & -1.943189 & -0.357492 \\ \mathrm{H} & 1.607786 & -2.134425 & -0.076136 \\ \mathrm{H} & 2.446824 & 1.185584 & 2.075693 \\ \mathrm{H} & 1.711951 & -0.437779 & 2.108541 \\ \mathrm{H} & 3.482153 & -0.251065 & 1.875135 \\ \mathrm{C} & -3.167646 & -0.366955 & 0.200123 \\ \mathrm{C} & -1.320966 & 1.897621 & -0.458357 \\ \mathrm{H} & -0.315626 & 2.261328 & -0.669183 \\ \mathrm{H} & -1.742272 & 2.413590 & 0.402077 \\ \mathrm{H} & -1.958210 & 2.016528 & -1.332416 \\ \mathrm{~N} & -4.335295 & -0.492069 & 0.328921\end{array}$
$\underset{16}{\operatorname{model}}$ system 29 last_Te_O_CN_mp2_bsse_freq.log.xyz

$\begin{array}{lrrr}\text { Te } & -1.601283 & 0.206309 & -0.000043 \\ \mathrm{O} & 2.449664 & 0.110211 & -0.000036 \\ \mathrm{C} & -0.757660 & -0.983071 & 1.556071 \\ \mathrm{C} & -0.757661 & -0.983701 & -1.555675 \\ \mathrm{H} & -1.030283 & -0.530669 & 2.510552 \\ \mathrm{H} & -1.161390 & -1.993910 & 1.506823 \\ \mathrm{H} & 0.326853 & -0.999585 & 1.453126 \\ \mathrm{H} & -1.030284 & -0.531685 & -2.510340 \\ \mathrm{H} & 0.326852 & -1.000172 & -1.452724 \\ \mathrm{H} & -1.161390 & -1.994520 & -1.506018 \\ \mathrm{C} & 3.657703 & -0.358101 & 0.000059 \\ \mathrm{C} & 2.380875 & 1.567020 & -0.000280 \\ \mathrm{H} & 1.317621 & 1.790380 & -0.000329 \\ \mathrm{H} & 2.866144 & 1.951014 & -0.897826 \\ \mathrm{H} & 2.866123 & 1.951313 & 0.897150 \\ \mathrm{~N} & 4.744233 & -0.814974 & 0.000151\end{array}$

model system 30 last_Te_S_CN_mp2_bsse_freq.log.xyz 


$\begin{array}{llcr}\text { Te } & -1.685489 & 0.287683 & -0.000086 \\ \mathrm{~S} & 2.117486 & -0.083268 & -0.000104 \\ \mathrm{C} & -1.341338 & -1.123978 & 1.559474 \\ \mathrm{C} & -1.341379 & -1.124917 & -1.558803 \\ \mathrm{H} & -1.475580 & -0.609247 & 2.511828 \\ \mathrm{H} & -2.062978 & -1.936857 & 1.482676 \\ \mathrm{H} & -0.323042 & -1.506348 & 1.493318 \\ \mathrm{H} & -1.475634 & -0.610756 & -2.511463 \\ \mathrm{H} & -0.323085 & -1.507258 & -1.492437 \\ \mathrm{H} & -2.063024 & -1.937743 & -1.481502 \\ \mathrm{C} & 3.764921 & -0.493868 & 0.000085 \\ \mathrm{C} & 2.294213 & 1.730674 & -0.000254 \\ \mathrm{H} & 1.271948 & 2.108460 & -0.000394 \\ \mathrm{H} & 2.819167 & 2.052963 & -0.897893 \\ \mathrm{H} & 2.818981 & 2.053131 & 0.897434 \\ \mathrm{~N} & 4.902914 & -0.808720 & 0.000220\end{array}$

\section{model system 31 last_Te_Se_CN_mp2_bsse_freq.log.xyz 16}

$\begin{array}{lrrr}\mathrm{Te} & -1.933148 & 0.281558 & -0.123550 \\ \mathrm{Se} & 1.749899 & -0.112268 & -0.047617 \\ \mathrm{C} & -1.779645 & -0.469378 & 1.865371 \\ \mathrm{C} & -1.697095 & -1.634998 & -1.024818 \\ \mathrm{H} & -1.886134 & 0.373022 & 2.550140 \\ \mathrm{H} & -2.578228 & -1.187916 & 2.047983 \\ \mathrm{H} & -0.803798 & -0.933228 & 2.008235 \\ \mathrm{H} & -1.723464 & -1.500994 & -2.106948 \\ \mathrm{H} & -0.737369 & -2.064029 & -0.736406 \\ \mathrm{H} & -2.514860 & -2.286737 & -0.718640 \\ \mathrm{C} & 3.554175 & -0.472108 & 0.124575 \\ \mathrm{C} & 1.981977 & 1.814533 & -0.260339 \\ \mathrm{H} & 0.972696 & 2.204506 & -0.388736 \\ \mathrm{H} & 2.586279 & 2.009394 & -1.143798 \\ \mathrm{H} & 2.444270 & 2.224952 & 0.634940 \\ \mathrm{~N} & 4.701614 & -0.727306 & 0.238296\end{array}$

\section{model system 32 MS_J12.xyz 16}

$\begin{array}{lrrr}\mathrm{Te} & 2.091218 & 0.248940 & -0.221539 \\ \mathrm{Te} & -1.486071 & -0.168575 & -0.038816 \\ \mathrm{C} & 1.983289 & -1.859021 & -0.507941 \\ \mathrm{C} & 2.203947 & 0.105637 & 1.901682 \\ \mathrm{H} & 1.862336 & -2.045919 & -1.575562 \\ \mathrm{H} & 2.903755 & -2.323774 & -0.156107 \\ \mathrm{H} & 1.124036 & -2.259520 & 0.031455 \\ \mathrm{H} & 2.262929 & 1.118148 & 2.303036 \\ \mathrm{H} & 1.307086 & -0.385141 & 2.279510 \\ \mathrm{H} & 3.097575 & -0.450212 & 2.184124 \\ \mathrm{C} & -3.520832 & -0.365756 & 0.201083 \\ \mathrm{C} & -1.684936 & 1.929447 & -0.370342 \\ \mathrm{H} & -0.683026 & 2.304084 & -0.578215 \\ \mathrm{H} & -2.091899 & 2.399050 & 0.522933 \\ \mathrm{H} & -2.336597 & 2.092228 & -1.226536 \\ \mathrm{~N} & -4.686090 & -0.498539 & 0.343847\end{array}$




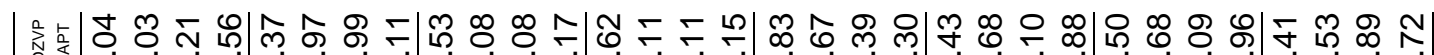
ヘ ш

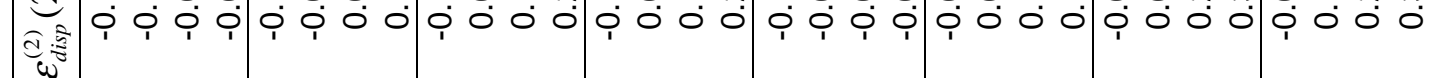

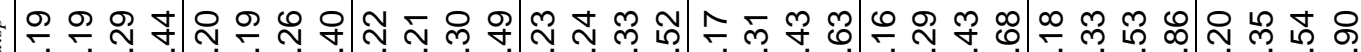
零

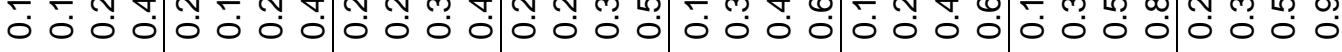

ลิ 过

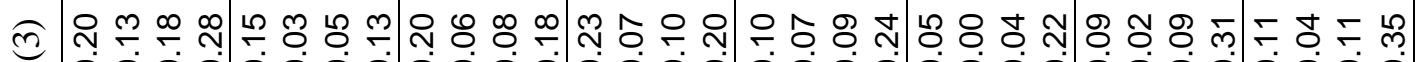
こ

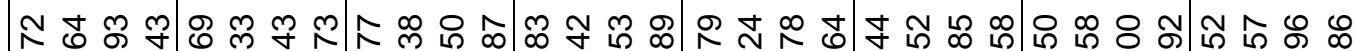

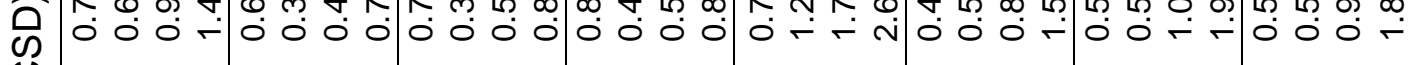


Figure FS1 Comparison of the interaction energy $E_{\text {int }}[\mathrm{kcal} / \mathrm{mol}]$ for model system 1 - 16 (a) and 17 32; (b) calculated by the MP2/cc-pVTZ-ECP, MP2/DGDZVP and the CCSD(T)/DGDZVP levels of theory.

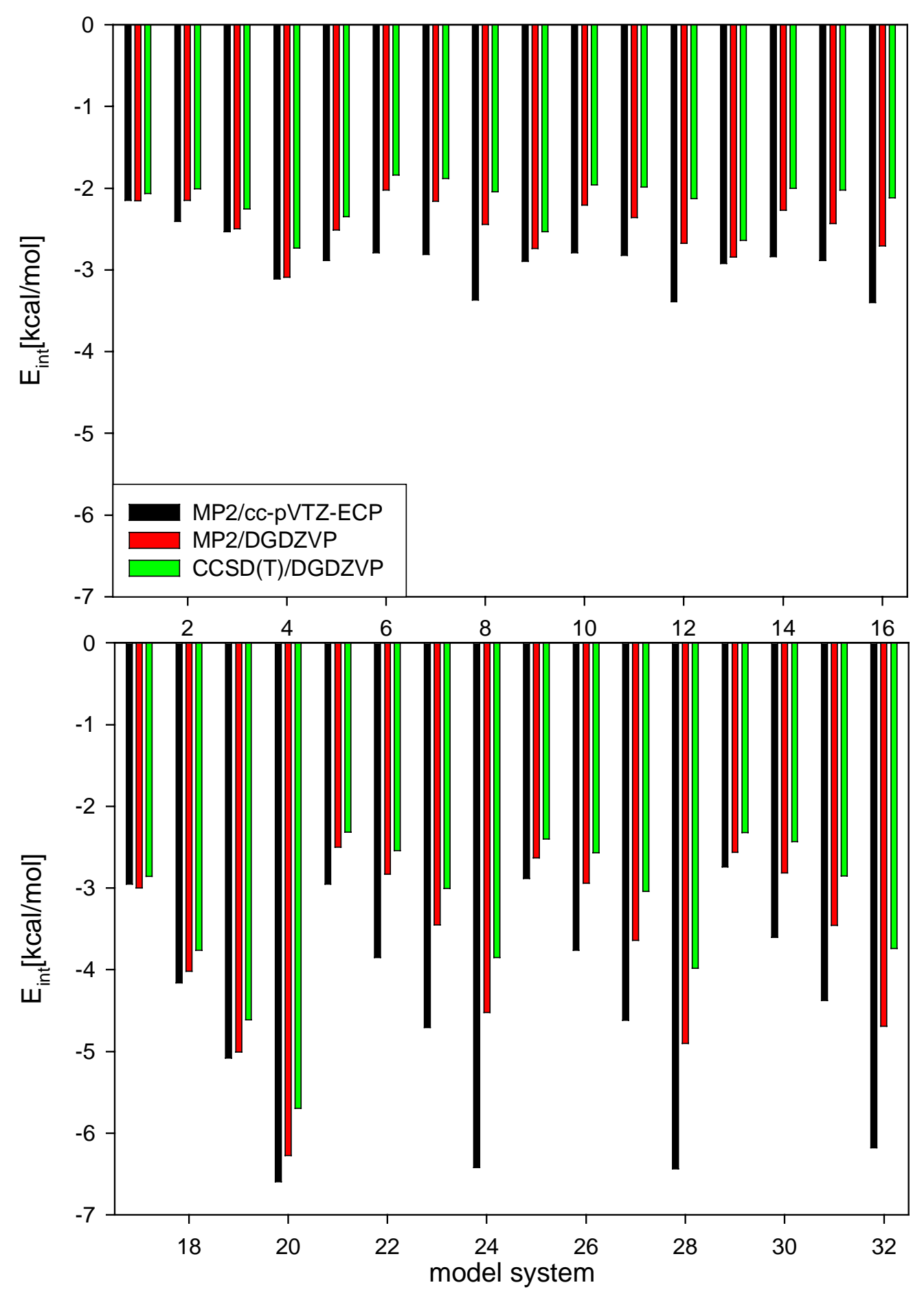


Figure FS2 Comparison of the interaction energy for model system 1 - 16 (a) and 17 - 32; (b) calculated by the SAPT algorithm using the $6-311 \mathrm{G}^{* *}$ and the DGDZVP basis sets on the MP2/cc-pVTZ-ECP optimized geometries.

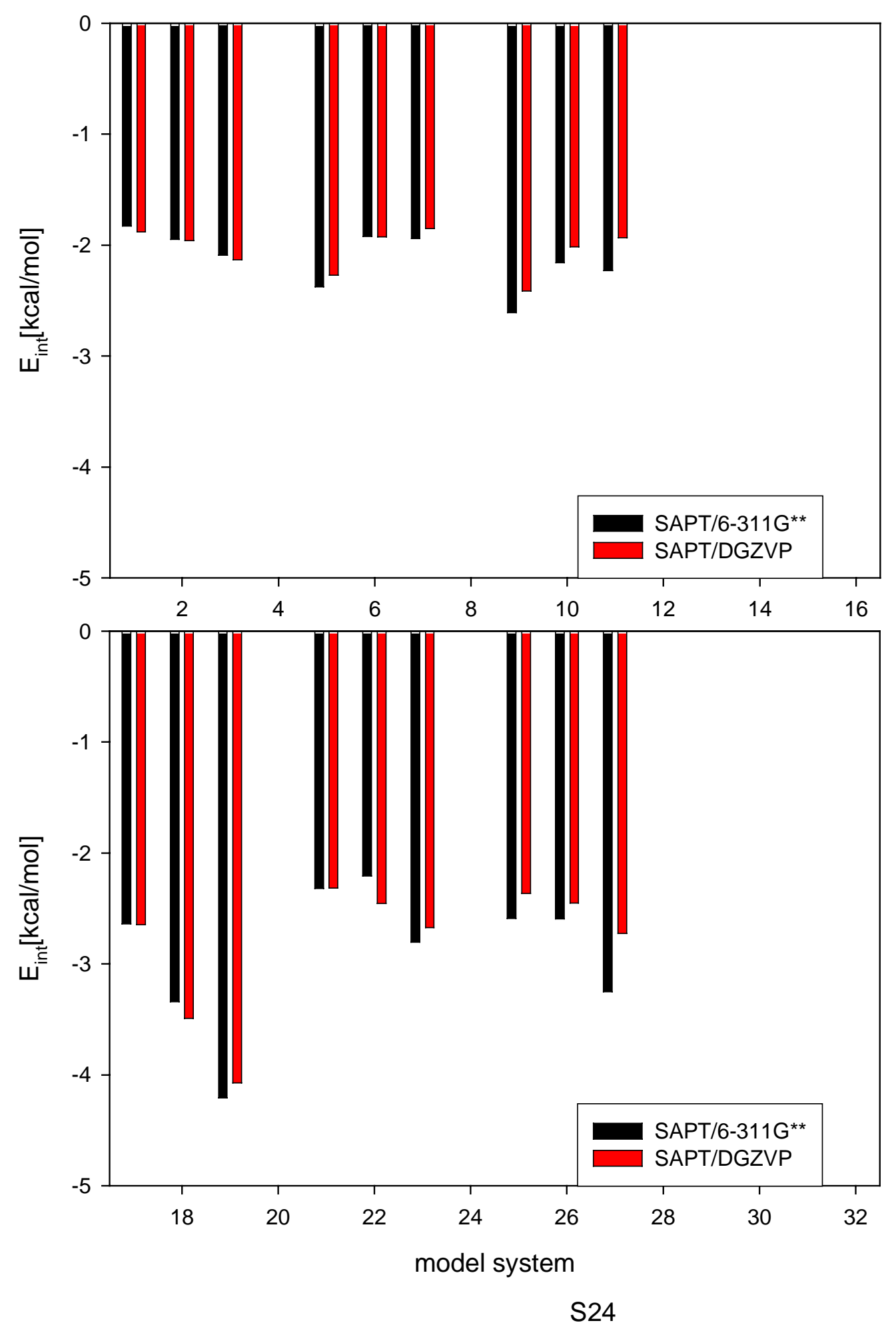


Figure FS3 Comparison of the electrostatic contribution to the interaction energy for model system 1 - 16 (a) and 17 - 32 (b) calculated by the SAPT algorithm using the $6-311 G^{* *}$ and the DGDZVP basis sets on the MP2/cc-pVTZ-ECP optimized geometries.
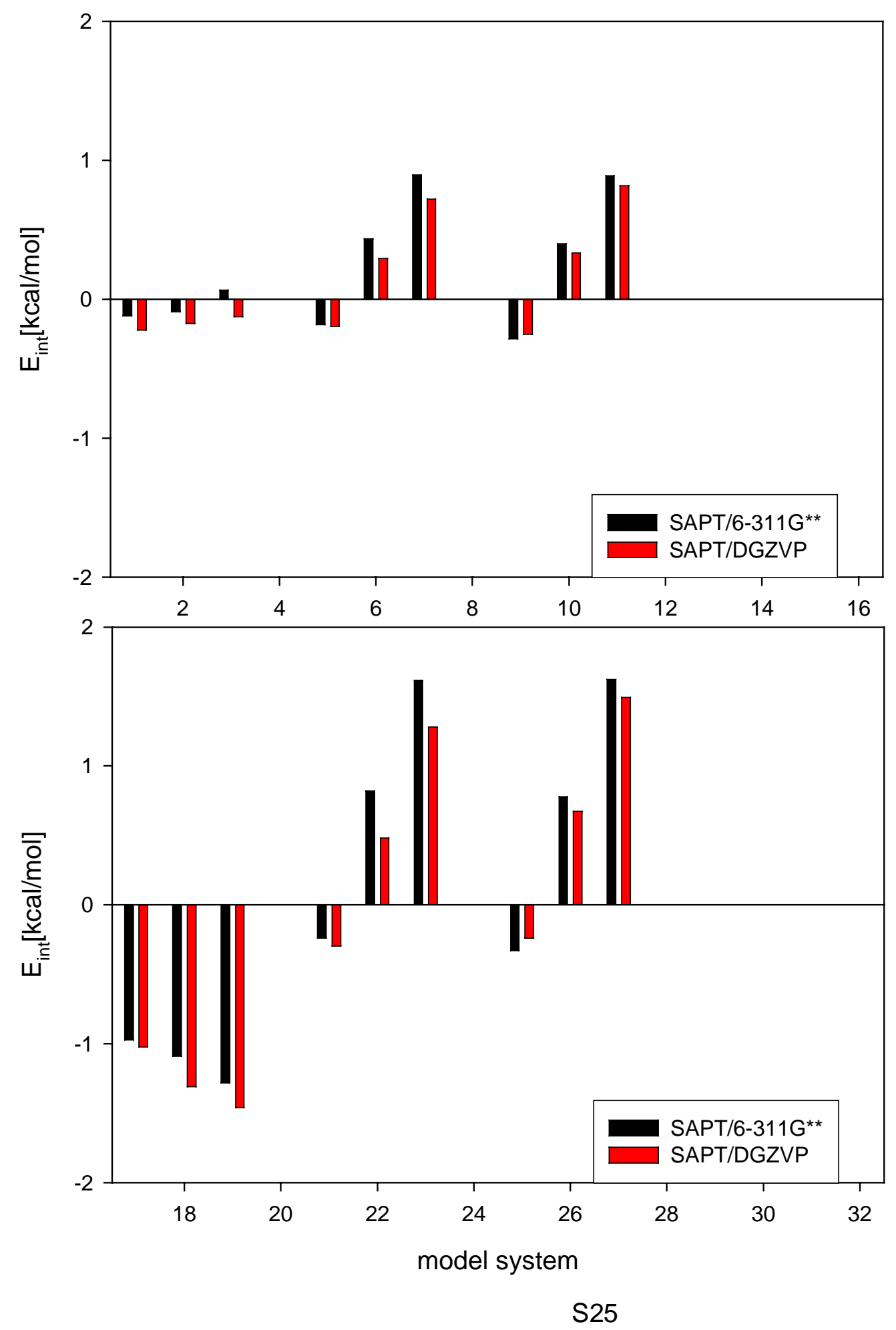
Figure FS4 Comparison of the induction contribution to the interaction energy for model system 1 16 (a) and 17 - 32 (b) calculated by the SAPT algorithm using the 6-311G** and the DGDZVP basis sets on the MP2/cc-pVTZ-ECP optimized geometries.

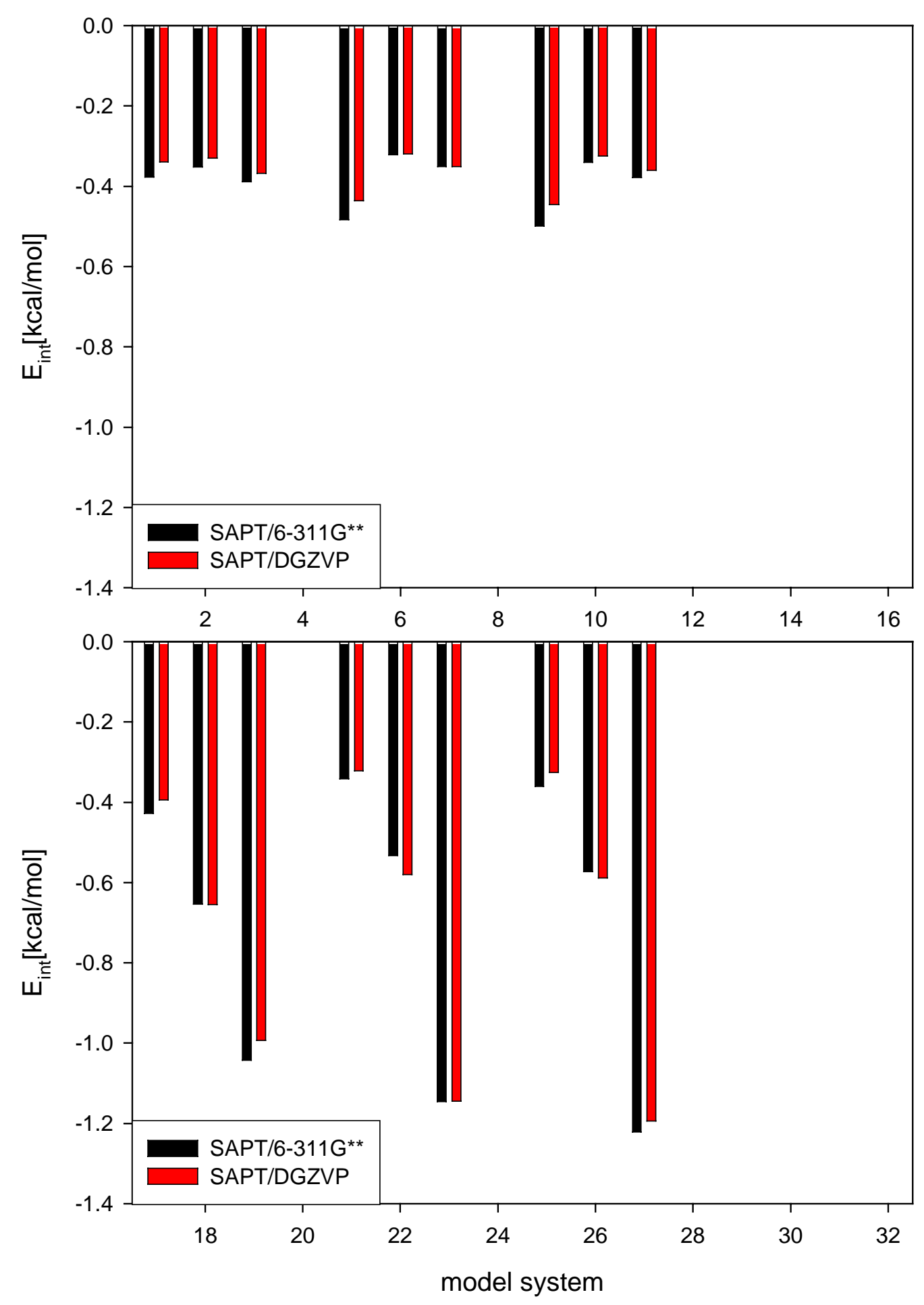


Figure FS5 Comparison of the dispersion contribution to the interaction energy for model system 1 16 (a) and 17 - 32 (b) calculated by the SAPT algorithm using the 6-311G** and the DGDZVP basis sets on the MP2/cc-pVTZ-ECP optimized geometries.

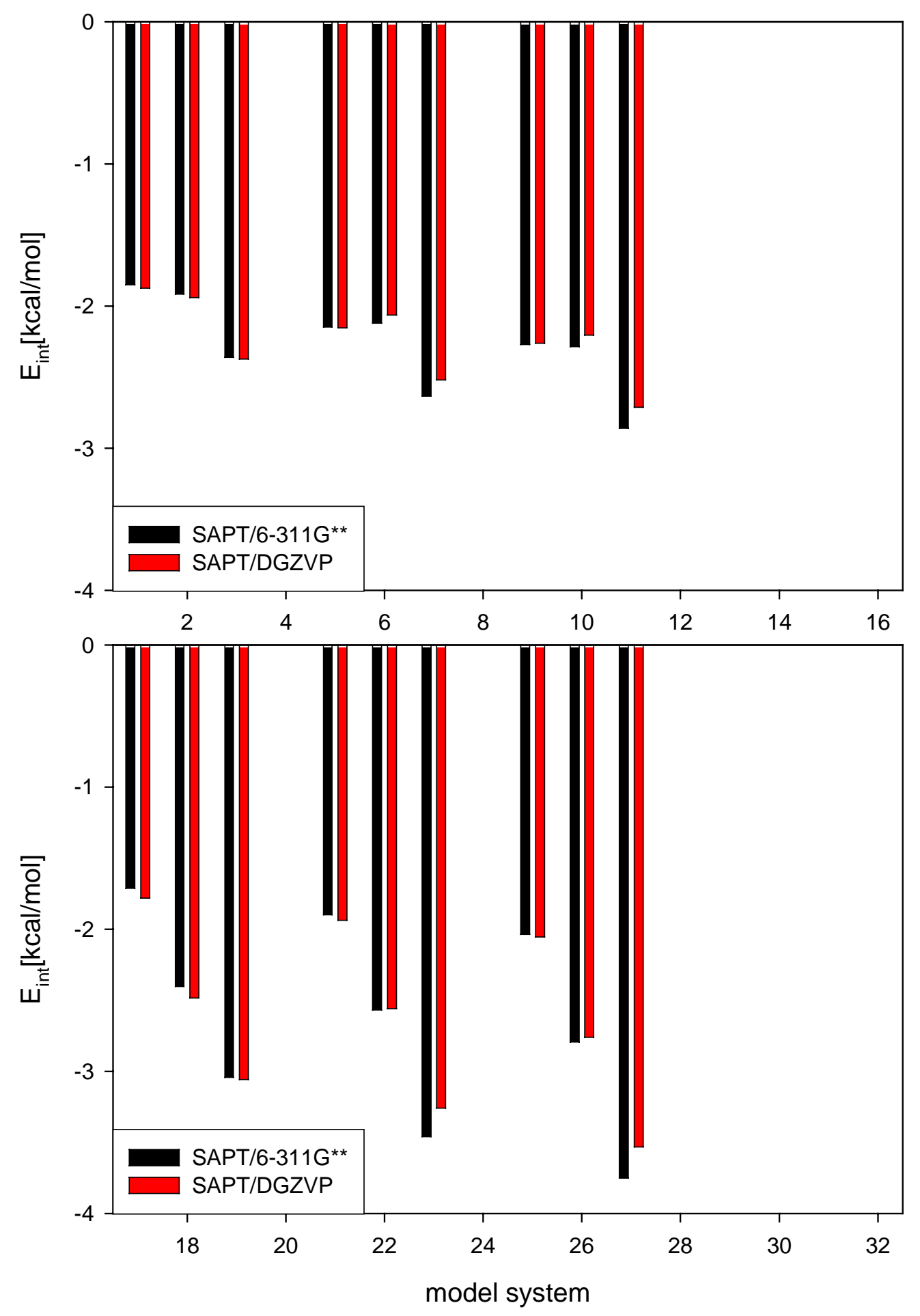


Figure FS6 Comparison of the exchange-correlation component of the interaction energy for model system 1 - 16 (a) and 17 - 32 (b) calculated by the SAPT algorithm using the $6-311 \mathrm{G}^{\star *}$ and the DGDZVP basis sets on the MP2/cc-pVTZ-ECP optimized geometries.

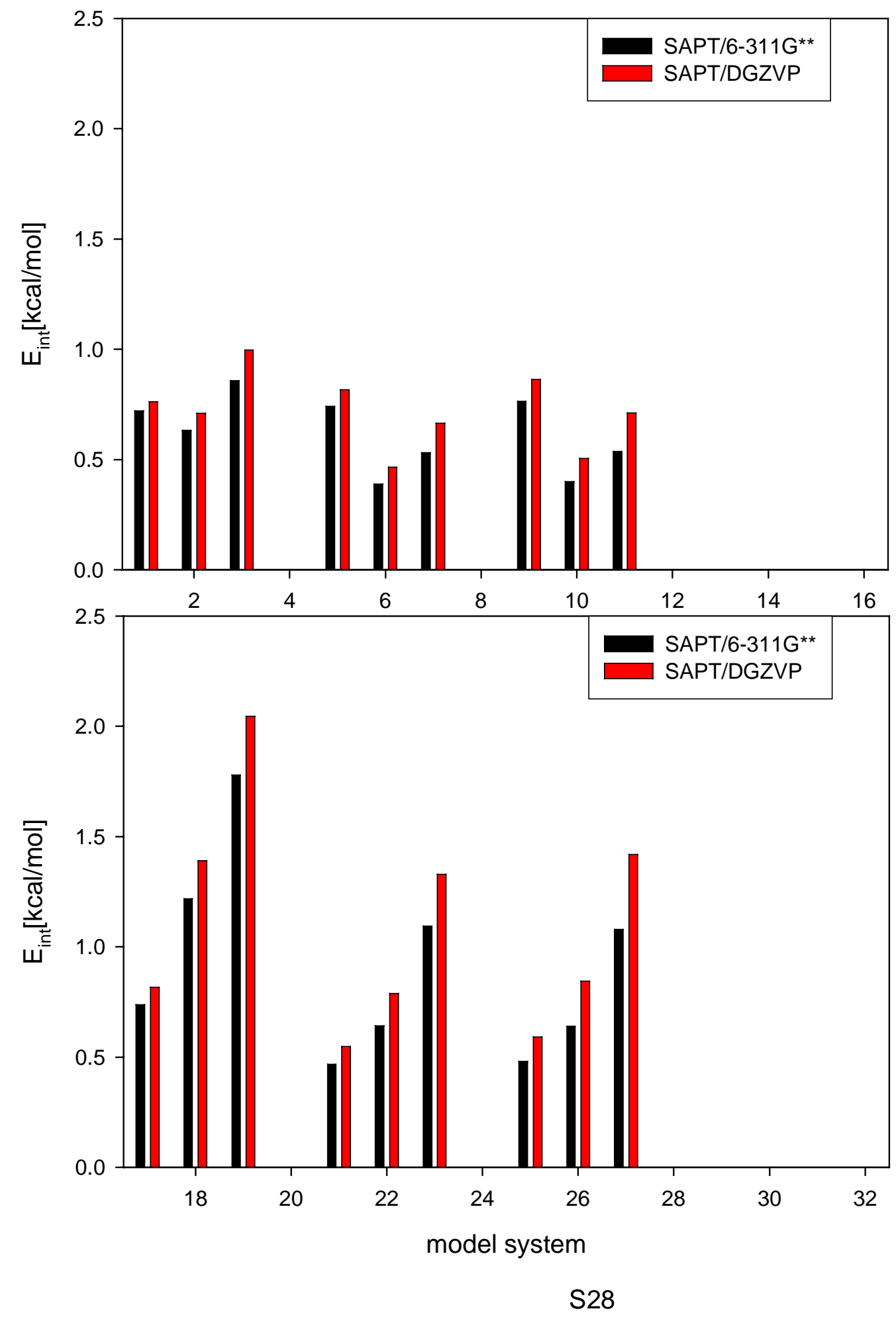


Figure FS7 Comparison of the interaction energies $E_{\text {int,SAPT }}^{\mathrm{DGDZVP}}$ and $\mathrm{E}_{\mathrm{int}, \mathrm{MP} 2}^{\mathrm{cC}-\mathrm{DVZ}}{ }^{\mathrm{ECP}}[\mathrm{kcal} / \mathrm{mol}]$ for model system 1 - 16 (a) and 17 - 32 (b) on the MP2/DGDZVP and MP2/cc-pVTZ-ECP optimized geometries, respectively.

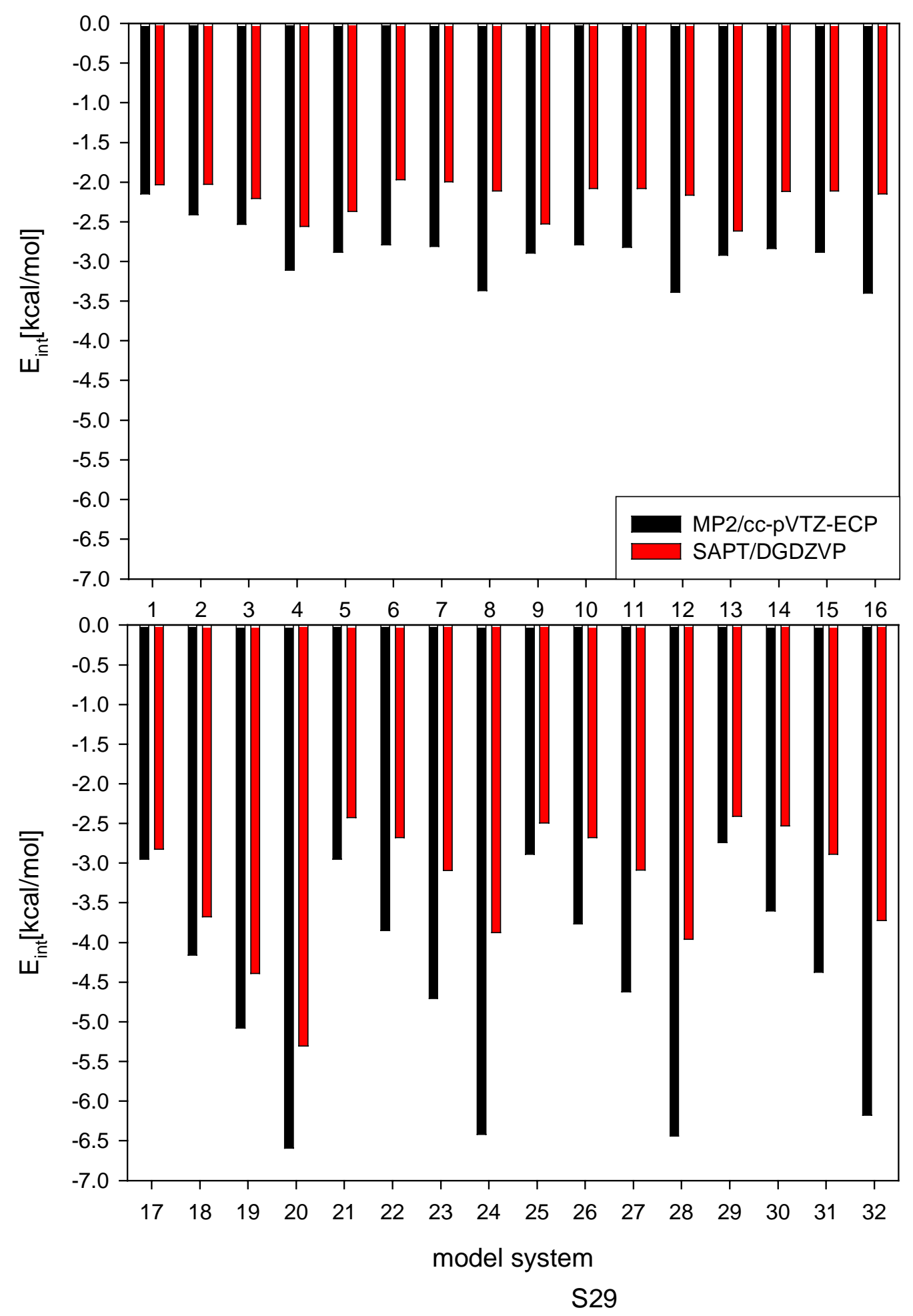

